\title{
Levantamento fitossociológico em Pastagens Degradadas sob CONDIÇÕES DE VÁRZEA ${ }^{1}$
}

\author{
Phyto-sociological Assessment of Degraded Pastures under Flooded Low Land Conditions
TUFFI SANTOS, L.D. ${ }^{2}$, SANTOS, I.C. ${ }^{3}$, OLIVEIRA, C.H. ${ }^{4}$, SANTOS, M.V..$^{5}$, FERREIRA, F.A. ${ }^{6}$ e QUEIROZ, D.S. ${ }^{3}$

\begin{abstract}
RESUMO - O levantamento foi realizado em duas áreas de várzea, eventualmente inundáveis, localizadas na Fazenda Experimental de Leopoldina, da Empresa de Pesquisa Agropecuária de Minas Gerais. A área 1, de 3 ha, estava ocupada por uma pastagem de capim-angola (Brachiaria mutica), mal manejada e sem controle de plantas daninhas há mais de dez anos. A área 2, de 5 ha, estava ocupada por uma pastagem de capim-setária (Setaria anceps cv. Kazungula), implantada na estação chuvosa do ano anterior, cuja formação ficou prejudicada pelo baixo estabelecimento da forrageira. Para o estudo fitossociológico, utilizou-se o método do quadrado inventário, aplicado por meio de um quadrado de $1,0 \mathrm{~m}^{2}$, lançado ao acaso 19 vezes na área 1 e 41 vezes na área 2 . As espécies encontradas foram identificadas e cadastradas. Na pastagem de capim-angola foram identificadas 27 espécies, distribuídas em 11 famílias e na pastagem de capim-setária 34 espécies distribuídas em 13 famílias. As famílias mais representativas em número de espécies foram: Poaceae (11), Asteraceae (6), Papilionoideae (5), Malvaceae e Euphorbiaceae (4). As maiores freqüencias foram das seguintes espécies: Cynodon dactylon, Sida rhombifolia, Cyperus esculentus, Mimosa pudica, Senna occidentalis, Setaria anceps cv. Kazungula e Paspalum urvillei. Em geral, as duas áreas apresentaram-se infestadas com plantas daninhas, inclusive com plantas tóxicas, espinescentes e de baixa palatabilidade, reduzindo a capacidade de suporte animal dos pastos e impedindo o aproveitamento adequado das áreas pelos bovinos.
\end{abstract}

Palavras-chave: fitossociologia, pastagem, plantas daninhas.

ABSTRACT - An assessment was carried out of two contingently flooded low land areas, located at the Experimental Farm of Leopoldina, owned by Empresa de Pesquisa Agropecuária de Minas Gerais (EPAMIG) - Brazil - MG. Area 1 (3 ha) was cultivated with Angola-grass (Brachiaria mutica) pasture, not well managed and without any weed control for more than 10 years. Area 2 (5 ha) was occupied by Setaria grass (Setaria anceps cv. Kazungula) pasture which had been established in the previous rainy season, with forage being affected by the slow growth of the plants. For the phyto-sociological study, the inventory square method was applied through a square of $1,0 \mathrm{~m}^{2}$, randomly placed 19 times in area 1 and 41 times in area 2. The species found were identified and recorded. In the Angola-grass pasture, 27 species were identified, distributed in 11 families; in the Setaria grass pasture, 34 species were identified, distributed in 13 families. The most representative families in number of species were: Poaceae (11); Asteraceae (6); Papilionoideae (5); Malvaceae and Euphorbiaceae (4). The most frequent species were: Cynodon dactylon, Sida rhombifolia, Cyperus esculentus, Mimosa pudica, Senna occidentalis, Setaria anceps cv. Kazungula and Paspalum urvillei. In general, both areas were infested with weeds, including toxic, spinescent and rather unpalatable plants, reducing the animal support capacity of the pastures and preventing adequate utilization of the areas by the cattle.

Key words: phyto-sociology, pasture, weeds.

Recebido para publicação em 3.3.2004 e na forma revisada em 10.9.2004.

2 Estudante de Doutorado em Fitotecnia, Dep. de Fitotecnia da Universidade Federal de Viçosa - DFT/UFV, 36570-000 Viçosa-MG; ${ }^{3}$ Pesquisador(a), EPAMIG/CTZM, 36570-000 Viçosa-MG. ${ }^{4}$ Engenheiro-Agrônomo; ${ }^{5}$ Estudante de Mestrado - DFT/UFV; ${ }^{6}$ Professor Titular, Dep. de Fitotecnia - DET-UFV.

Planta Daninha, Viçosa-MG, v. 22, n. 3, p. 343-349, 2004 


\section{INTRODUÇÃO}

O Brasil possui o maior rebanho bovino comercial do mundo, com 170 milhões de cabeças; é o segundo maior produtor de carne bovina, tendo potencial de ampliar significativamente sua produção. Em geral, a produção pecuária brasileira, seja ela de corte ou de leite, tem as pastagens como base de sustentação. Segundo Paulino et al. (2002), as pastagens representam uma fonte de alimento de menor custo, eficiente energeticamente e de acordo com a demanda da sociedade, que exige que a qualidade e o modo de produção da carne e do leite atendam as exigências de preservação dos recursos ambientais e de sustentabilidade dos sistemas de produção.

Diante do contexto de uma pecuária competitiva e empresarial, a pastagem assume papel de importância para o sucesso da atividade, sendo necessária a sua manutenção em condições adequadas de produção. Entretanto, o que se observa na prática é a predominância de pastagens degradadas, o que pode ser considerado um dos principais problemas do sistema de produção de bovinos. Estima-se que $80 \%$ dos quase 60 milhões de hectares das áreas de pastagens na região de cerrados apresentam algum estádio de degradação (Macedo et al., 2000). A degradação pode ser causada por diversos fatores, entre eles a falta de adaptação ao meio da espécie cultivada, a má formação inicial, a perda da fertilidade do solo pela falta de adubação de manutenção e o manejo inadequado, incluindo o superpastejo.

Um dos problemas resultantes da degradação pelo manejo inadequado das pastagens é a infestação por plantas daninhas, que, devido à sua capacidade de interferência, reduz a produtividade das forrageiras. Ao competir pelos fatores de crescimento, as plantas daninhas promovem queda da capacidade de suporte da pastagem, aumentam o tempo de formação e de recuperação do pasto, podem causar ferimentos e/ou intoxicação aos animais e comprometem a estética da propriedade (Rosa, 2001; Silva et al., 2002; Pereira \& Silva, 2000).

O levantamento fitossociológico em pastagem é importante na obtenção do conhecimento sobre as populações e a biologia das espécies encontradas, constituindo uma importante ferramenta no embasamento técnico de recomendações de manejo e tratos culturais, seja para implantação, recuperação ou condução das pastagens. Vários são os trabalhos que buscam o conhecimento da flora invasora em pastagem, nas mais diversas regiões (Peixoto et al., 1982; Silva \& Dias Filho, 2001; Modesto Júnior \& Mascarenhas, 2001; Lara et al., 2003).

Grande número de plantas daninhas é citado como infestantes em pastagens, e as famílias mais importantes são: Leguminoseae, Gramineae, Malvaceae, Myrtaceae, Cyphaceae, Asteraceae, Cyperaceae, Rubiaceae e Labiateae (Silva \& Dias Filho, 2001; Lara et al., 2003).

Este trabalho foi proposto com o objetivo de efetuar o levantamento fitossociológico em duas áreas de várzea sob pastejo por bovinos.

\section{MATERIAL E MÉTODOS}

O trabalho foi realizado em duas áreas de várzea eventualmente inundáveis, na Fazenda Experimental de Leopoldina, da Empresa de Pesquisa Agropecuária de Minas Gerais (EPAMIG). O município de Leopoldina está localizado na Zona da Mata, Estado de Minas Gerais, a 220 metros de altitude, $21^{\circ} 31^{\prime} 50$ " latitude sul e $42^{\circ} 38^{\prime} 30^{\prime \prime}$ longitude oeste; possui clima tropical úmido (megatérmico) de savana, com inverno seco e verão chuvoso, com precipitação pluvial de $1.200 \mathrm{~mm}$. A temperatura média do mês mais frio é superior a $18{ }^{\circ} \mathrm{C}$ (Antunes, 1986).

A área experimental incluiu dois pastos degradados, implantados em solo de várzea, sob pastejo por bovinos. As duas áreas são sujeitas a eventuais inundações, normalmente de curta duração, apresentando problemas de drenagem em condições de excesso de chuva. A área 1, de três hectares, estava estabelecida com capim-angola (Brachiaria mutica), em uso há mais de dez anos, sem uma estratégia de manejo definida; os principais fatores determinantes da degradação foram a falta de controle de espécies invasoras, o superpastejo por bovinos, principalmente durante a estação seca, e a falta de adubação de manutenção. A área 2, com cinco hectares, que se apresentava quase totalmente tomada por invasoras, foi submetida a aração e calagem 
em setembro de 2000; em outubro foi realizada gradagem e semeadura de capim-setária (Setaria anceps cv. Kazungula) a lanço, sem adubação, na densidade de $3 \mathrm{~kg}$ de sementes puras viáveis por hectare, segundo o valor cultural informado pelo fornecedor. O pasto não se estabeleceu adequadamente, sendo observada baixa germinação das sementes de setária. Ademais, o mau estabelecimento do capim-setária pode ser atribuído ao seu desenvolvimento inicial lento e à falta de controle de plantas daninhas, levando a uma infestação de espécies competidoras.

O levantamento foi realizado entre os meses de setembro e novembro de 2001, e as pastagens encontravam-se sob pastejo por vacas em lactação. Para o estudo fitossociológico, utilizou-se o método do quadrado inventário, aplicado por meio de um quadrado de 1,0 m de lado, lançado ao acaso 19 vezes na pastagem de capim-angola e 41 vezes na de capim-setária, perfazendo um total de 60 parcelas e uma área amostral de $60 \mathrm{~m}^{2}$. A cada lançamento, as espécies encontradas dentro do quadrado foram colhidas e devidamente identificadas e cadastradas, sendo obtido o número de indivíduos por espécie.

Para análise das comunidades das espécies presentes, foram calculados os parâmetros fitossociológicos: freqüência - permite avaliar a distribuição das espécies nas parcelas; densidade - quantidade de plantas de cada espécie por unidade de área; abundância - informa sobre a concentração das espécies na área; freqüência relativa, densidade relativa e abundância relativa - informam a relação de cada espécie com as outras espécies encontradas na área; e índice de valor de importância - indica quais espécies são mais importantes dentro da área estudada.

No cálculo dessas características foram utilizadas as fórmulas que se seguem (Mueller-Dombois \& Ellenberg, 1974):

$$
\begin{aligned}
& \text { Frequiência }(\text { Fre })=\frac{\mathrm{N}^{\mathrm{o}} \text { de parcelas que contêm a espécie }}{\mathrm{N}^{\mathrm{o}} \text { total de parcelas utilizadas }} \\
& \text { Densidade }(\mathrm{Den})=\frac{\mathrm{N}^{\mathrm{o}} \text { total de indivíduos por espécie }}{\text { Área total amostrada }} \\
& \text { Abundância }(\mathrm{Abu})=\frac{\mathrm{N}^{\mathrm{o}} \text { total de indivíduos por espécie }}{\mathrm{N}^{\mathrm{o}} \text { total de parcelas que contêm a espécie }}
\end{aligned}
$$

Freqüência Relativa $\left(\right.$ Frr) $=\frac{\text { Freqüência da espécie x } 100}{\text { Freqüência total de todas as espécies }}$

Densidade Relativa $($ Der $)=\frac{\text { Densidade da espécie x } 100}{\text { Densidade total das espécies }}$

Abundância Relativa $(\mathrm{Abr})=\frac{\text { Abundância da espécie x } 100}{\text { Abundância total de todas as espécies }}$

Índice de Valor de Importäncia $($ IVI $)=$ Frr + Der + Abr

Para avaliação da similaridade entre as populações botânicas nas duas áreas estudadas, foi utilizado o IS - Índice de Similaridade de Sorense (Sorense, 1972), através da fórmula:

Índice de Similaridade $(\%)=(2 \mathrm{a} / \mathrm{b}+\mathrm{c}) * 100$

em que: $\mathrm{a}=$ número de espécies comuns às duas áreas; b e c = número total de espécies nas duas áreas comparadas. O IS varia de 0 a 100, sendo máximo quando todas as espécies são comuns às duas áreas e mínimo quando não existem espécies em comum.

\section{RESULTADOS E DISCUSSÃO}

As espécies encontradas, suas respectivas famílias e os parâmetros fitossociológicos calculados para cada área estão apresentados nas Tabelas 1 e 2 .

Foram identificadas 42 espécies, distribuídas em 14 famílias botânicas; na pastagem de capim-angola foram encontradas 27 espécies, distribuídas em 11 famílias (Tabela 1), e na pastagem de capim-setária, 34 espécies, distribuídas em 13 famílias (Tabela 2). A família mais representativa em número de espécies foi a Poaceae, com um total de 11, seguida pelas famílias Compositae (6), Papilionoideae (5), Malvaceae e Euphorbiaceae (4) (Tabelas 1 e 2).

Dentro da família Poaceae, além das espécies daninhas encontradas, também figuram outras espécies de interesse forrageiro, além daquelas introduzidas nas áreas para formação do pasto, como Paspalum conjugatum, Paspalum maritimum e Paspalum notatum (Tabelas 1 e 2). Essas espécies são comuns em áreas de várzea com problemas de drenagem e, juntamente com aquelas utilizadas na formação da pastagem nas suas respectivas áreas, também são consumidas pelos bovinos. Outra espécie que

Planta Daninha, Viçosa-MG, v. 22, n. 3, p. 343-349, 2004 
Tabela 1 - Espécies dentro de cada família encontradas na pastagem de capim-angola e suas respectivas características fitossociológicas no período de setembro a novembro de 2001. Leopoldina-MG

\begin{tabular}{|c|c|c|c|c|c|c|c|c|c|}
\hline Espécie & NTI & NPP & DEN & FRE & $\mathrm{ABU}$ & DER & FRR & ABR & IVI \\
\hline \multicolumn{10}{|c|}{ Malvaceae } \\
\hline Sida rhombifolia $\mathrm{L}$. & 831 & 14 & 43,74 & 0,74 & 59,36 & 25,91 & 11,11 & 11,79 & 48,82 \\
\hline Sida urens L. & 4 & 2 & 0,21 & 0,11 & 2,00 & 0,12 & 1,59 & 0,40 & 2,11 \\
\hline Urena lobata $\mathrm{L}$. & 3 & 1 & 0,16 & 0,05 & 3,00 & 0,09 & 0,79 & 0,60 & 1,48 \\
\hline \multicolumn{10}{|c|}{ Poaceae } \\
\hline Brachiaria mutica (Forsk.) Stapf & 788 & 13 & 41,47 & 0,68 & 60,62 & 24,57 & 10,32 & 12,04 & 46,93 \\
\hline Paspalum notatum Fluegge. & 366 & 9 & 19,26 & 0,47 & 40,67 & 11,41 & 7,14 & 8,08 & 26,64 \\
\hline Paspalum conjugatum Berg. & 139 & 1 & 7,32 & 0,05 & 139,00 & 4,33 & 0,79 & 27,61 & 32,74 \\
\hline Cynodon dactylon (L.) Pers. & 98 & 3 & 5,16 & 0,16 & 32,67 & 3,06 & 2,38 & 6,49 & 11,93 \\
\hline Paspalum maritimum Trin. & 90 & 6 & 4,74 & 0,32 & 15,00 & 2,81 & 4,76 & 2,98 & 10,55 \\
\hline Eleusine indica (L.) Gaertn. & 56 & 3 & 2,95 & 0,16 & 18,67 & 1,75 & 2,38 & 3,71 & 7,84 \\
\hline Paspalum urvillei Steud. & 33 & 10 & 1,74 & 0,53 & 3,30 & 1,03 & 7,94 & 0,66 & 9,62 \\
\hline \multicolumn{10}{|c|}{ Cyperaceae } \\
\hline Cyperus esculentus L. & 496 & 12 & 26,11 & 0,63 & 41,33 & 15,47 & 9,53 & 8,21 & 33,20 \\
\hline \multicolumn{10}{|c|}{ Euphorbiaceae } \\
\hline Croton lundianus Muell Arg. & 91 & 5 & 4,79 & 0,26 & 18,20 & 2,84 & 3,97 & 3,62 & 10,42 \\
\hline Chamaesuce prostrata Small. & 6 & 3 & 0,32 & 0,16 & 2,00 & 0,19 & 2,38 & 0,40 & 2,97 \\
\hline \multicolumn{10}{|c|}{ Compositae } \\
\hline Elephantopus mollis H.P. \& K. & 43 & 9 & 2,26 & 0,47 & 4,78 & 1,34 & 7,14 & 0,95 & 9,43 \\
\hline Eupatorium pauciflorum Rafin. & 3 & 1 & 0,16 & 0,05 & 3,00 & 0,09 & 0,79 & 0,60 & 1,48 \\
\hline Conyza canadensis L. & 2 & 2 & 0,11 & 0,11 & 1,00 & 0,06 & 1,59 & 0,20 & 1,85 \\
\hline Erechitites hieraciifolius Rafin. & 2 & 1 & 0,11 & 0,05 & 2,00 & 0,06 & 0,79 & 0,40 & 1,25 \\
\hline \multicolumn{10}{|c|}{ Papilionoideae } \\
\hline Desmodium adscendens DC. & 34 & 2 & 1,79 & 0,11 & 17,00 & 1,06 & 1,59 & 3,38 & 6,02 \\
\hline Desmodium tortuosum Webb. & 26 & 6 & 1,37 & 0,32 & 4,33 & 0,81 & 4,76 & 0,86 & 6,43 \\
\hline Centrosema arenarium Benth. & 14 & 2 & 0,74 & 0,11 & 7,00 & 0,44 & 1,59 & 1,39 & 3,41 \\
\hline Centrosema pubecens Benth. & 2 & 2 & 0,11 & 0,11 & 1,00 & 0,06 & 1,59 & 0,20 & 1,85 \\
\hline \multicolumn{10}{|c|}{ Commelinaceae } \\
\hline Commelina diffusa Burn $\mathrm{f}$. & 28 & 2 & 1,47 & 0,11 & 14,00 & 0,87 & 1,59 & 2,78 & 5,24 \\
\hline \multicolumn{10}{|c|}{ Mimoseae } \\
\hline Mimosa pudica Linn. & 25 & 7 & 1,32 & 0,37 & 3,57 & 0,78 & 5,56 & 0,71 & 7,05 \\
\hline \multicolumn{10}{|c|}{ Caesalpinoideae } \\
\hline Senna occidentalis Link. & 13 & 4 & 0,68 & 0,21 & 3,25 & 0,41 & 3,18 & 0,65 & 4,23 \\
\hline \multicolumn{10}{|c|}{ Caryophyllaceae } \\
\hline Drymaria cordata A. Gray, & 8 & 2 & 0,42 & 0,11 & 4,00 & 0,25 & 1,59 & 0,79 & 2,63 \\
\hline \multicolumn{10}{|c|}{ Solanaceae } \\
\hline Solanum palinacanthum Dun. & 5 & 3 & 0,26 & 0,16 & 1,67 & 0,16 & 2,38 & 0,33 & 2,87 \\
\hline Solanum americanum Mill. & 1 & 1 & 0,05 & 0,05 & 1,00 & 0,03 & 0,79 & 0,20 & 1,02 \\
\hline Total & 3.207 & 126 & 168,79 & 6,63 & 503,41 & 100 & 100 & 100 & 300 \\
\hline
\end{tabular}

$\mathrm{NTI}=$ número total de indivíduos, $\mathrm{NPP}=$ número de parcelas presentes, $\mathrm{DEN}=$ densidade, $\mathrm{FRE}=$ freqüência $, \mathrm{ABU}=\mathrm{abundância}, \mathrm{DER}=$ densidade relativa, $\mathrm{FRR}=$ frequiência relativa, $\mathrm{ABR}=$ abundância relativa e IVI = índice de valor de importância. 
Tabela 2 - Espécies dentro de cada família encontradas na pastagem de capim-setária (área 2) e suas respectivas características fitossociológicas no período de setembro a novembro de 2001, Leopoldina-MG

\begin{tabular}{|c|c|c|c|c|c|c|c|c|c|}
\hline Espécie & NTI & NPP & DEN & FRE & $\mathrm{ABU}$ & DER & FRR & $\mathrm{ABR}$ & IVI \\
\hline \multicolumn{10}{|c|}{ Malvaceae } \\
\hline Sida rhombifolia $\mathrm{L}$. & 8.488 & 40 & 207,02 & 0,98 & 212,20 & 55,50 & 15,63 & 28,99 & 100,13 \\
\hline Sida cordifolia $\mathrm{L}$. & 4 & 3 & 0,10 & 0,07 & 1,33 & 0,03 & 1,17 & 0,18 & 1,38 \\
\hline Sida urens $\mathrm{L}$. & 1 & 1 & 0,02 & 0,02 & 1,00 & 0,01 & 0,39 & 0,14 & 0,53 \\
\hline \multicolumn{10}{|c|}{ Poaceae } \\
\hline Cynodon dactylon (L.) Pers. & 3.463 & 16 & 84,46 & 0,39 & 216,44 & 22,64 & 6,25 & 29,57 & 58,47 \\
\hline Setaria sphacelata Stapf. ex. Massey cv. kazungula & 602 & 29 & 14,68 & 0,71 & 20,76 & 3,94 & 11,34 & 2,84 & 18,11 \\
\hline Brachiariamutica (Forsk.) Stapf. & 204 & 2 & 4,98 & 0,05 & 102,00 & 1,33 & 0,78 & 13,93 & 16,05 \\
\hline Digitaris horizontalis Willd. & 53 & 3 & 1,29 & 0,07 & 17,67 & 0,35 & 1,17 & 2,41 & 3,93 \\
\hline Sorghum halepense (L.) Pers. & 24 & 4 & 0,59 & 0,10 & 6,00 & 0,16 & 1,56 & 0,82 & 2,54 \\
\hline Eleusine indica (L.) Gaertn. & 23 & 9 & 0,56 & 0,22 & 2,56 & 0,15 & 3,52 & 0,35 & 4,02 \\
\hline Paspalum urvillei Steud. & 15 & 9 & 0,37 & 0,22 & 1,67 & 0,10 & 3,52 & 0,23 & 3,84 \\
\hline Paspalum conjugatum Berg. & 13 & 2 & 0,32 & 0,05 & 6,50 & 0,09 & 0,78 & 0,89 & 1,75 \\
\hline Dactyloctenium aegyptium P. Beauv. & 1 & 1 & 0,02 & 0,02 & 1,00 & 0,01 & 0,39 & 0,14 & 0,53 \\
\hline \multicolumn{10}{|c|}{ Cyperaceae } \\
\hline Cyperus esculentus $\mathrm{L}$. & 1.801 & 23 & 43,93 & 0,56 & 78,30 & 11,78 & 8,99 & 10,70 & 31,46 \\
\hline \multicolumn{10}{|c|}{ Mimosoideae } \\
\hline Mimosa pudica Linn. & 350 & 39 & 8,54 & 0,95 & 8,97 & 2,29 & 15,24 & 1,23 & 18,76 \\
\hline Mimosa debilis Humb \& Bonpl. ex. Willd. & 1 & 1 & 0,02 & 0,02 & 1,00 & 0,01 & 0,39 & 0,14 & 0,53 \\
\hline \multicolumn{10}{|c|}{ Caesalpinoideae } \\
\hline Senna occidentalis Link. & 74 & 19 & 1,80 & 0,46 & 3,89 & 0,48 & 7,43 & 0,53 & 8,44 \\
\hline \multicolumn{10}{|c|}{ Papilionoideae } \\
\hline Desmodium adscendens DC. & 56 & 3 & 1,37 & 0,07 & 18,67 & 0,37 & 1,17 & 2,55 & 4,09 \\
\hline Centrosema arenarium Benth. & 47 & 12 & 1,15 & 0,29 & 3,92 & 0,31 & 4,69 & 0,54 & 5,53 \\
\hline Centrosema pubecens Benth. & 2 & 1 & 0,05 & 0,02 & 2,00 & 0,01 & 0,39 & 0,27 & 0,68 \\
\hline Aeschynomene rudis Benth. & 1 & 1 & 0,02 & 0,02 & 1,00 & 0,01 & 0,39 & 0,14 & 0,53 \\
\hline \multicolumn{10}{|c|}{ Euphorbiaceae } \\
\hline Chamaesuce prostrata Small. & 27 & 6 & 0,66 & 0,15 & 4,50 & 0,18 & 2,35 & 0,61 & 3,14 \\
\hline Croton lundianus Muell Arg. & 4 & 4 & 0,10 & 0,10 & 1,00 & 0,03 & 1,56 & 0,14 & 1,73 \\
\hline Asclepias curassavica L. & 1 & 1 & 0,02 & 0,02 & 1,00 & 0,01 & 0,39 & 0,14 & 0,53 \\
\hline Chamaesuce hirta (L.) Millsp. & 1 & 1 & 0,02 & 0,02 & 1,00 & 0,01 & 0,39 & 0,14 & 0,53 \\
\hline \multicolumn{10}{|c|}{ Compositae } \\
\hline Agerantum conizoides Linn. & 16 & 9 & 0,39 & 0,22 & $\begin{array}{l}1,78 \\
1,7\end{array}$ & 0,10 & 3,52 & 0,24 & 3,87 \\
\hline Eupatorium pauciflorum Rafin. & 3 & 2 & 0,07 & 0,05 & 1,50 & 0,02 & 0,78 & 0,20 & 1,01 \\
\hline Eclipta Alba Kuntze. & 3 & 1 & 0,07 & 0,02 & 3,00 & 0,02 & 0,39 & 0,41 & 0,82 \\
\hline Conyza canadensis $\mathrm{L}$. & 1 & 1 & 0,02 & 0,02 & 1,00 & 0,01 & 0,39 & 0,14 & 0,53 \\
\hline \multicolumn{10}{|c|}{ Solanaceae } \\
\hline Solanum americanum Mill. & 10 & 6 & 0,24 & 0,15 & 1,67 & 0,07 & 2,35 & 0,23 & 2,64 \\
\hline \multicolumn{10}{|c|}{ Commelinaceae } \\
\hline Commelina diffusa Burn $\mathrm{f}$. & 9 & 2 & 0,22 & 0,05 & 4,50 & 0,06 & 0,78 & 0,61 & 1,46 \\
\hline \multicolumn{10}{|c|}{ Labiatae } \\
\hline Leonurus sibiricus $\mathrm{L}$. & 3 & 2 & 0,07 & 0,05 & 1,50 & 0,02 & 0,78 & 0,20 & 1,01 \\
\hline Leonotis nepetaefolia Schimp. ex Benth. & 1 & 1 & 0,02 & 0,02 & 1,00 & 0,01 & 0,39 & 0,14 & 0,53 \\
\hline \multicolumn{10}{|c|}{ Onagraceae } \\
\hline Ludwigia leptocarpa Hara. & 1 & 1 & 0,02 & 0,02 & 1,00 & 0,01 & 0,39 & 0,14 & 0,53 \\
\hline \multicolumn{10}{|c|}{ Amaranthaceae } \\
\hline Amaranthus spinosus L. & 1 & 1 & 0,02 & 0,02 & 1,00 & 0,01 & 0,39 & 0,14 & 0,53 \\
\hline Total & 15.304 & 256 & 373,30 & 6,20 & 732,30 & 100 & 100 & 100 & 300 \\
\hline
\end{tabular}

$\mathrm{NTI}=$ número total de indivíduos, $\mathrm{NPP}=$ número de parcelas presentes, $\mathrm{DEN}=$ densidade, $\mathrm{FRE}=$ freqüência $, \mathrm{ABU}=\mathrm{abundância}, \mathrm{DER}=$ densidade relativa, $\mathrm{FRR}$ = frequiência relativa, $\mathrm{ABR}$ = abundância relativa e IVI = índice de valor de importância. 
merece destaque nas duas áreas é a gramaseda ou grama-de-burro (Cynodon dactylon), que constitui uma alternativa de forrageira tropical para alimentação de rebanhos leiteiros (Alvim et al., 1997). Na pastagem de capimsetária, o capim-angola, espécie que caracteriza a outra área estudada, também aparece expressivamente, com IVI =16. Alvim et al. (1992) apontam o capim-angola como a principal espécie espontânea das áreas de várzea da Zona da Mata de Minas Gerais e a classifica como uma alternativa viável para alimentar vacas mestiças em lactação durante todo o ano. Contudo, não apresenta qualidade suficiente para sustentar alta produção de leite em vacas especializadas, devido às suas qualidades nutricionais.

Nas duas áreas também estão presentes espécies dos gêneros Desmodium, Centrosema e Aeschynomene, da família Leguminoseae (subfamília Papilionoideae), que são forrageiras capazes de fixar nitrogênio biologicamente, podendo ser consumidas pelos animais, principalmente no período seco do ano, contribuindo para melhorar a dieta bovina e aumentar o consumo de forragem. Segundo Serrão \& Falesi (1977), o gênero Desmodium é o de maior importância entre as leguminosas encontradas nas pastagens cultivadas, que servem para o pastejo dos animais.

A espécie Senna ocidentalis, popularmente conhecida como fedegoso, foi encontrada nas duas áreas, com IVI de 4,10 e 7,65 para as pastagens de capim-angola e capim-setária, respectivamente (Tabelas 1 e 2). O fedegoso é uma planta considerada tóxica para bovinos, eqüinos, ovinos e caprinos, devendo ser erradicada das áreas. Seu efeito é cumulativo nos animais, sendo os eqüinos mais sensíveis que os bovinos, podendo se intoxicar com o consumo de 1,5 a 3,0 g por quilo de peso vivo (Melo \& Oliveira, 2000). A malícia (Mimosa pudica) é outra espécie que merece destaque, em razão da grande quantidade de espinhos em seus ramos, que podem impedir o pastejo nas suas proximidades ou mesmo ferir o animal. São comuns ferimentos nas tetas de vacas em lactação causados por plantas espinescentes, o que pode prejudicar a ordenha. A pastagem de capim-setária apresentou maior infestação pela malícia, sendo a quarta espécie de maior importância na área, com IVI $=18,76$
(Tabela 2); já na pastagem de capim-angola a malícia apresentou IVI = 7,05 (Tabela 1).

As espécies mais representativas na pastagem de capim-angola (Tabela 1) e seus respectivos IVIs foram: Sida rhombifolia $(48,82)$, Brachiaria mutica $(46,93)$ e Cyperus esculentus $(33,20)$. Na pastagem de capimsetária (Tabela 2), as espécies mais representativas e seus respectivos IVI foram: Sida rhombifolia $(100,13)$, Cynodon dactylon $(58,47)$ e Cyperus esculentus $(31,46)$.

O Índice de Similaridade (IS) entre as duas áreas é de 62,30\%, indicando uma similaridade mediana. Esta similaridade é explicada por se tratar de áreas vizinhas e sob as mesmas condições ambientais, podendo as diferenças ser atribuídas, em parte, à ação antrópica. $\mathrm{Na}$ pastagem de capim-angola, a intervenção do homem se limitou à exploração da área para pastejo, ainda que sem critérios de manejo ajustados ao potencial da área e sem manutenção, enquanto a área de capim-setária foi recentemente exposta à implantação de uma forrageira e, conseqüentemente, ao manejo do solo e tratos culturais necessários. Assim, as operações de aração e gradagem na área de implantação do capim-setária podem ter influenciado o banco de sementes viáveis, favorecendo a germinação de maior número de espécies nesta área, o que não ocorreu na pastagem de capim-angola.

Junto com a formação apropriada da pastagem - pela escolha adequada da espécie forrageira, preparo do solo, semeadura na época, quantidade e profundidade recomendada, correção e adubação de acordo com a exigência da forrageira -, o manejo da pastagem estabelecida é um dos métodos mais importantes no controle de plantas daninhas. $\mathrm{O}$ método e a intensidade de pastejo devem estar ajustados às características morfofisiológicas da forrageira e ao nível de investimento adotado, principalmente em relação às adubações de manutenção. As práticas de manejo devem propiciar a rápida recuperação da forrageira após pastejo ou corte, favorecendo seu poder de competição com as plantas daninhas.

Em geral, as duas áreas apresentaram-se infestadas com plantas daninhas, inclusive com espécies tóxicas, espinescentes e de baixa palatabilidade, reduzindo a capacidade 
de suporte animal dos pastos e impedindo o aproveitamento adequado das áreas pelos bovinos.

\section{LITERATURA CITADA}

ALVIM, M. J.; SIMÃO NETO, M.; DUSI, G. A. Efeito da disponibilidade de forragem e da adubação em pastagem de capim-angola sobre a produção de leite. Pesq. Agropec. Bras., v. 27, n. 11, p. 1541-1550, 1992.

ALVIM, M. J. et al. Efeito de dois níveis de concentrado sobre a produção de leite de vacas da raça Holandesa em pastagem de "coast-cross" (Cynodon dactylon (L.) Pers.). R. Soc. Brás. Zootec., v. 26, n. 5, p. 967-975, 1997.

ANTUNES, F. Z. Caracterização climática do Estado de Minas Gerais. Inf. Agropec., v.12, n. 138, p. 9-13, 1986.

LARA, J. F. R.; MACEDO, J. F.; BRANDÃO, M. Plantas daninhas em pastagens de várzeas do estado de Minas Gerais. Planta Daninha, v. 21, n. 1, p. 11-20, 2003.

MELO, M. M.; OLIVEIRA, N. J. F. Plantas tóxicas. Belo Horizonte: 2000. p. 7-76. (Cadernos Técnicos de Veterinária e Zootecnia, 32).

MACEDO, M. C. M.; RICHEL, A. N.; ZIMMER, A. H. Z. Degradação e alternativas de recuperação e renovação de pastagens. Campo Grande: EMBRAPA - MCNPGC, 2000. 4 p. (Comunicado Técnico 62).

MODESTO JÚNIOR, M. S.; MASCARENHAS, R. E. B. Levantamento da infestação de plantas daninhas associada a uma pastagem cultivada de baixa produtividade no nordeste paraense. Planta Daninha, v. 19, n. 1, p. 11-21, 2001.

MUELLER-DOMBOIS, D.; ELLENBERG, H. A. Aims and methods of vegetation ecology. New York: John Wiley, 1974. $547 \mathrm{p}$.
PAULINO, N. F. et al. Bovinocultura de ciclo curto em pastagens. In: SIMPÓSIO DE PRODUÇÃO DE GADO DE CORTE - SICORTE, 3., 2002, Viçosa. Anais... Viçosa: SBZ, 2002. p. 153-196.

PEIXOTO, A. L.; CARVALHO, S. M.; DA ROSA, M. M. T. Análise botânica de um campo de pastagem no estado do Rio de Janeiro. Planta Daninha, v. 5, n. 2, p. 1-7, 1982.

PEREIRA J. R. P.; SILVA, W. Controle de plantas daninhas em pastagens. Instrução técnica para o produtor de leite. Juiz de Fora: EMBRAPA/2000.

ROSA, B. Influência do uso de herbicidas na recuperação de pastagens de capim-braquiarão. J. Propasto Goiás, v. 4, n. 1, 2001.

SERRÃO, E. A. S.; FALESI, I. C. Pastagens do trópico úmido brasileiro. In: SIMPÓSIO SOBRE MANEJO DE PASTAGENS, 4., Piracicaba: ESALQ, 1977.

SILVA, A. A.; WERLANG, R. C.; FERREIRA, L. R. Controle de plantas daninhas em pastagens. In: SIMPÓSIO SOBRE MANEJO ESTRATÉGICO DA PASTAGEM, 1., 2002, Viçosa. Anais...Viçosa: SBZ, 2002. p. 273-310.

SILVA, D. S. M.; DIAS FILHO, M. B. Banco de sementes de plantas daninhas em solo cultivado com pastagens de Brachiaria brizantha e Brachiaria humidicola de diferentes idades. Planta Daninha, v. 19, n. 2, p. 179-185, 2001.

SORENSE, T. A method of stablishing groups of equal amplitude in plant society based on similarity of species content. In: ODUN, E. P. (Ed.). Ecologia. 3.ed. México: Interamericana, 1972. $640 \mathrm{p}$. 